\title{
Mortalidad por enfermedades huérfanas en Colombia, 2008-2013
}

\author{
Julio César Martínez ${ }^{1}$, Sandra Patricia Misnaza² \\ 1 Centro de Investigación y Atención en Salud del Magdalena Medio, CISMAG, Barrancabermeja, Colombia \\ 2 FETP Colombia-Grupo de Epidemiología Aplicada, Instituto Nacional de Salud, Bogotá, D.C., Colombia
}

Introducción. Las enfermedades huérfanas caracterizadas por su baja prevalencia, comúnmente son de origen genético y degenerativo, y amenazan la vida.

Objetivo. Describir la mortalidad por enfermedades huérfanas y analizar la tendencia en Colombia entre 2008 y 2013.

Materiales y métodos. Se trata de un estudio descriptivo. Se analizó la tendencia de las tasas de mortalidad a partir de los certificados de defunción entre el 2008 y el 2013. Se calcularon las tasas específicas de mortalidad y las ajustadas por edad y sexo.

Resultados. Se atribuyeron 7.135 defunciones a enfermedades huérfanas; 51,4 \% ocurrieron en hombres de todas las edades. La tasa media de mortalidad fue de 2,53 muertes por 100.000 personas. La tendencia mostró un patrón de ascenso, aunque muy heterogéneo, en el territorio nacional. Las mayores tasas de mortalidad por 100.000 habitantes se registraron en Bogotá $(20,0)$, la región Andina $(5,3)$ y la Caribe $(3,7)$. Las principales causas de muerte en hombres fueron: leucemia linfoblástica aguda, distrofia muscular, displasia broncopulmonar originada en el periodo perinatal, esclerosis múltiple, síndrome de Guillain-Barré y gastrosquisis, y en mujeres, esclerosis múltiple, leucemia linfoide aguda, gastrosquisis, displasia broncopulmonar originada en el periodo perinatal, síndrome de Guillain-Barré y leucemia mieloide aguda. La tasa media de mortalidad por leucemia linfoblástica aguda fue de 0,17 por 100.000 hombres menores de 15 años, y la de mortalidad por esclerosis múltiple fue de 0,16 en mujeres mayores de 40 años.

Conclusiones. Las causas de muerte mostraron un comportamiento similar en ambos sexos; sin embargo, la mayor carga de mortalidad se registró en la población masculina de todas edades en Bogotá.

Palabras clave: registros de mortalidad, enfermedades raras, leucemia linfoide, esclerosis múltiple, salud pública, Colombia.

doi: https://doi.org/10.7705/biomedica.v38i0.3876

\section{Rare diseases mortality in Colombia, 2008-2013}

Introduction: Rare diseases are characterized by their low prevalence, often of genetic origin, degenerative and life threatening.

Objective: To describe mortality by orphan diseases and to analyze its trends in Colombia from 2008 to 2013.

Materials and methods: We conducted a descriptive study to analyze mortality rate trends from the death certificates between 2008 and 2013. We calculated specific mortality rates and adjusted by age and sex.

Results: Seven thousand one hundred and thirty five deaths were attributed to orphan diseases, and $51.4 \%$ of them occurred among men of all ages. The mean mortality rate during the study period was 2.53 deaths per 100,000 people. Overall, the trend showed an increasing pattern of mortality although very heterogeneous across the country. Mortality rates were higher in Bogotá (20), and the Andes and the Caribbean regions (5.3 and 3.7 deaths per 100,000 population). The five most important causes of mortality among men were: Acute lymphoblastic leukemia, muscular dystrophy, bronchopulmonary dysplasia originating in the perinatal period, multiple sclerosis, Guillain-Barré syndrome and gastroschisis, and among women: Multiple sclerosis, acute lymphoblastic leukemia, gastroschisis, bronchopulmonary dysplasia originating in the perinatal period, Guillain-Barré syndrome and acute myeloid leukemia. The mean mortality rate by acute lymphoblastic leukemia was 0.17 deaths per 100,000 men younger than 15 years and that of multiple sclerosis was 0.16 in women over 40 years of age.

\section{Contribución de los autores:}

Julio Cesar Martínez: solicitud de la información ante la entidad correspondiente

Ambos autores participaron en la consolidación y el análisis de la información, y en la escritura del artículo. 
Conclusion: The causes of death showed a similar pattern in both sexes. However, the burden of mortality was higher among men of all ages in Bogota.

Key words: Mortality registries; rare diseases; leukemia, lymphoid; multiple sclerosis; public health; Colombia.

doi: https://doi.org/10.7705/biomedica.v38i0.3876

Las enfermedades huérfanas constituyen un grupo amplio y variado de trastornos que afectan a un reducido número de personas o a una proporción baja de la población general. Según el Ministerio de Salud y Protección Social de Colombia, en el mundo se han identificado entre 6.000 y 7.000 enfermedades huérfanas (1).

Los países de la Unión Europea consideran como enfermedades huérfanas a aquellas que afectan a 1 de cada 2.000 personas; Estados Unidos, como las que afectan a menos de 200.000 personas; Japón, como las que afectan a un número inferior a 50.000 personas; Taiwán establece su prevalencia como inferior a 1 por cada 10.000 personas $y$, Colombia, en 1 por cada $5.000(2,3)$.

Este grupo de enfermedades difiere ampliamente en términos de gravedad, aunque, en promedio, la esperanza de vida de los pacientes se reduce significativamente, aunque el impacto y la expectativa de vida varían mucho de una enfermedad a otra. Algunos pacientes mueren al nacer, otros sufren enfermedades degenerativas que amenazan la vida, y otros padecen enfermedades que son compatibles con la vida si se diagnostican y manejan a tiempo y adecuadamente (4).

Las enfermedades huérfanas y raras son de baja prevalencia pero con una alta tasa de mortalidad. Por lo general, tienen una evolución crónica muy grave, con múltiples deficiencias motoras, sensoriales y cognitivas. Por lo tanto, suelen presentar gran complejidad clínica, lo que dificulta su reconocimiento y diagnóstico. Entre el 75 y el $80 \%$ de estas dolencias infrecuentes se deben a una alteración en los genes, y el 20 al $25 \%$ restante son enfermedades autoinmunitarias (5).

Entre los antecedentes normativos de Colombia, encontramos que en el año 2010 se expidió la Ley 1392, por medio de la cual se reconoce a las enfermedades huérfanas como de especial interés y se adoptan las normas tendientes a garantizar la

\section{Correspondencia:}

Julio César Martínez, Carrera 24S № 43-60, Barrancabermeja, Colombia

Teléfono: (577) 6124956

jucer1023@hotmail.com

Recibido: 25/04/17; aceptado: 07/09/17 protección social por parte del Estado colombiano a la población que las padece y a sus cuidadores (6). En el año 2013, mediante la Resolución 430, se estableció un listado integrado por 1.920 enfermedades, producto de la revisión bibliográfica sistemática de literatura y el análisis de la información disponible sobre las enfermedades huérfanas listadas en Orphanet (7), y en el 2015 se actualizó dicho listado, definiendo para Colombia 2.149 enfermedades huérfanas (8). En el 2017, el Instituto Nacional de Salud publicó en el protocolo de vigilancia en salud pública (2), los códigos de la Clasificación Internacional de Enfermedades en su décima versión (CIE-10) correspondientes a enfermedades huérfanas o raras, de acuerdo con la revisión de la codificación realizada por el Ministerio de Salud y Protección Social.

Se desconoce el impacto, la carga de morbilidad y mortalidad, los medios diagnósticos, los tratamientos y las repercusiones en cuanto a discapacidad, de muchas de las enfermedades huérfanas. Esto puede deberse a que, por su baja prevalencia, aún son nuevas para la comunidad científica. También, la dificultad para su análisis tiene que ver con que no todas las enfermedades huérfanas cuentan con un código ClE-10.

Debido a que este grupo de enfermedades suele presentar una alta tasa de mortalidad y al desconocimiento de las mismas en nuestro medio, el objetivo de este estudio fue describir el comportamiento de la mortalidad y analizar la tendencia en Colombia, durante el periodo comprendido entre el 2008 y el 2013, en consonancia con la Ley 1392 de 2010.

\section{Materiales y métodos}

Se hizo un estudio descriptivo para caracterizar la mortalidad por las enfermedades huérfanas en Colombia entre 2008 y 2013, a partir de los certificados individuales de defunción registrados en la base oficial de datos del Departamento Administrativo Nacional de Estadística (DANE), como única fuente secundaria de información.

Se revisó la Resolución 2048 de 2015, que listó y priorizó dichas enfermedades en Colombia. El listado inicial incluía 2.149 enfermedades huérfanas, 
las cuales no contaban con codificación CIE-10, por lo cual se revisó el anexo 1 del protocolo de vigilancia en salud pública del Instituto Nacional de Salud. En dicho anexo, se encontró que 897 enfermedades contaban con CIE-10; sin embargo, la gran mayoría eran códigos inespecíficos, por lo cual se hizo una nueva revisión código a código, estableciendo que 147 tenían códigos específicos o compatibles en el CIE-10, constituyéndose estos en los códigos por depurar en las bases de defunciones no fetales de las estadísticas vitales del DANE (anexo 1).

Se revisaron todas las causas de muerte del certificado de defunción (causa directa, causas antecedentes 1, 2 y 3, causas patológicas 1 y 2 y causa básica).

El universo incluyó 1'193.048 muertes registradas por todas las causas en las defunciones no fetales de 2008 a 2013. Tras la depuración de la base de datos, se analizaron 7.135 registros de defunción cuyas causas de muerte eran las enfermedades huérfanas, de los cuales se excluyeron aquellos en los que no se determinaba la edad y los de personas que residían en el extranjero.

Se evaluó la calidad de los datos con respecto a la integridad de las variables (completitud de los datos en los campos del certificado de defunción), evaluando la proporción de datos recopilados frente a aquellos sin información o sin diligenciar. Se incluyeron todas las defunciones cuyas variables de estudio cumplieran con una integridad de información superior al $85 \%$. La integridad promedio de celdas vacías superó el 99,9\% en todas las variables analizadas; en cuanto a casillas sin información, la integridad superó el 99,7 \% en la mayoría de las variables, excepto en el nivel educativo, cuyo resultado fue de $87 \%$.

Mediante un análisis univariado, se caracterizó el comportamiento de las defunciones registradas según las variables sociodemográficas: año de defunción, sexo, edad, departamento de residencia habitual, nivel educativo, tipo de afiliación al sistema general de seguridad social en salud, pertenencia étnica y códigos de causas de defunción.

Las muertes debidas a enfermedades huérfanas se analizaron en términos de frecuencias absolutas y relativas. Se estimaron las tasas de mortalidad específica por sexo y grupos de edad, y se expresaron por 100.000 personas al año. Como denominador, se empleó la población a mitad de periodo de los respectivos grupos de edad reportada por el DANE durante los años de estudio. Para comparar la evolución de la mortalidad en el tiempo, se calcularon las tasas anuales ajustadas por edad, utilizando el método directo (9) y tomando como población de referencia la población de Colombia por grupos de edad y por quinquenios según el censo de 2005. Las tasas de mortalidad se analizaron por tipo de enfermedad huérfana para los diez diagnósticos más frecuentes, por años de estudio y por sexo, y los diagnósticos restantes se agruparon en "otras enfermedades huérfanas".

El procesamiento de los datos y el análisis de la información se hicieron mediante el empleo de hojas de cálculo Microsoft Excel ${ }^{\mathrm{TM}}$ de 2013 y el paquete estadístico R Studio, versión 0.99.878.

\section{Consideraciones éticas}

En el diseño de este estudio, se contemplaron los principios metodológicos para salvaguardar el interés de la ciencia y el respeto a los derechos de las personas. Las bases de defunciones no contenían información sobre la identidad de los individuos; en los procedimientos realizados no se intervino ninguna persona, no se violó el derecho a la privacidad y los resultados se presentaron de manera agrupada.

\section{Resultados}

Entre el 2008 y el 2013, en Colombia se registraron 1'193.048 defunciones por todas las causas, de las cuales 7.135 presentaron, al menos, un diagnóstico de enfermedad huérfana como causa de muerte, en residentes de alguno de los 32 departamentos y 5 distritos de Colombia (cuadro 1).

Las defunciones por este grupo de enfermedades se presentaron con mayor frecuencia en los hombres, una proporción importante de las personas fallecidas no contaba con ningún grado de escolaridad y una alta proporción residía en

Cuadro 1. Distribución de muertes por enfermedades huérfanas, Colombia, 2008-2013

\begin{tabular}{cccc}
\hline Año & Defunciones $^{*}$ & $\begin{array}{c}\text { Enfermedades } \\
\text { huérfanas }\end{array}$ & $\%$ \\
\hline 2008 & 196.943 & 1.058 & 0,54 \\
2009 & 196.931 & 831 & 0,42 \\
2010 & 200.524 & 1.353 & 0,67 \\
2011 & 195.823 & 1.282 & 0,65 \\
2012 & 199.756 & 1.250 & 0,63 \\
2013 & 203.071 & 1.361 & 0,67 \\
Total & 1.193 .048 & 7.135 & 0,60 \\
\hline
\end{tabular}

** Defunciones por todas las causas

Fuente: DANE. Estadísticas vitales, Colombia 
la cabecera municipal. En relación con el tipo de afiliación al Sistema General de Seguridad Social en Salud (SGSSS), el 45,5\% pertenecía al régimen contributivo. De las defunciones registradas, el 6,5\% de la población se reconocía como afrocolombiana, afrodescendiente o mulata. En cuanto a los grupos de edad más afectados, se encontró una mayor proporción de fallecimientos en los extremos de la vida (cuadro 2).

La tasa media de mortalidad cruda para el periodo, fue de 2,53 por 100.000 personas; la tasa más alta se observó en el 2010 y se presentó una leve reducción a lo largo de los dos años siguientes, para volver a aumentar en el 2013 (figura 1).

La tasa media de mortalidad debida a enfermedades huérfanas según grupos de edad, mostró un comportamiento similar en ambos sexos, pero las tasas fueron mayores en la población masculina y en los extremos de la vida. Las tasas más altas se alcanzaron a partir de los 75 años (figura 2).

Según el tipo de enfermedades huérfanas, en general, en los hombres de todos los grupos de edad se registraron las mayores tasas de mortalidad. Sin embargo, se encontró que la leucemia linfoblástica aguda aportó la mayor en menores de 15 años, seguida por la distrofia muscular en el grupo de 15 a 19 años, la gastrosquisis, la displasia broncopulmonar originada en el periodo perinatal $y$, en adultos mayores de 45 años, la esclerosis múltiple y el síndrome de Guillain-Barré (cuadro 3).

En cuanto a las mujeres, las mayores tasas de esclerosis múltiple se registraron en mayores de 40 años y, las de leucemia linfoblástica aguda, en menores de 15 años, seguidas por las de la gastrosquisis y la displasia broncopulmonar originada en el periodo perinatal, las cuales presentaron un comportamiento similar en los hombres, aunque en términos generales fueron más bajas (cuadro 4).

Durante la primera infancia (menores de cinco años), las principales causas de muerte por enfermedades huérfanas fueron: displasia broncopulmonar originada en el periodo perinatal, gastrosquisis, holoprosencefalia, atresia de los conductos biliares, onfalocele (exónfalos), enfermedad de Hirschsprung, síndrome de dificultad respiratoria del recién nacido, malformaciones congénitas múltiples no clasificadas en otra parte, anomalía de Ebstein y estenosis congénita de la válvula tricúspide, las cuales correspondieron al 56,3\% de las muertes en menores de cinco años y al 16,4\% de todas las muertes por estas enfermedades.
Cuadro 2. Comportamiento de las características sociales y demográficas de las defunciones por enfermedades huérfanas, Colombia, 2008-2013

\begin{tabular}{|c|c|c|}
\hline Variable/Categoría & Casos & $\%$ \\
\hline \multicolumn{3}{|l|}{ Sexo } \\
\hline Masculino & 3.667 & 51,4 \\
\hline Femenino & 3.468 & 48,6 \\
\hline Total & 7.135 & 100 \\
\hline \multicolumn{3}{|l|}{ Nivel educativo } \\
\hline Sin escolaridad & 2.445 & 34,3 \\
\hline Básica primaria & 1.894 & 26,5 \\
\hline Básica secundaria & 840 & 11,8 \\
\hline Educación media & 512 & 7,2 \\
\hline Educación superior & 511 & 7,2 \\
\hline Sin información & 933 & 13,1 \\
\hline Total & 7.135 & 100 \\
\hline \multicolumn{3}{|l|}{ Régimen de afiliación al SGSSS } \\
\hline Contributivo & 3.246 & 45,5 \\
\hline Subsidiado & 3.060 & 42,9 \\
\hline Excepción & 226 & 3,2 \\
\hline Especial & 26 & 0,4 \\
\hline No afiliado & 508 & 7,1 \\
\hline Sin información & 69 & 1,0 \\
\hline Total & 7.135 & 100 \\
\hline \multicolumn{3}{|l|}{ Pertenencia étnica } \\
\hline Indígena & 107 & 1,5 \\
\hline Rom, gitano & 18 & 0,3 \\
\hline Raizal & 5 & 0,1 \\
\hline Palenquero & 5 & 0,1 \\
\hline Afrocolombiano, afrodescendiente, mulato & 465 & 6,5 \\
\hline Otros & 5.904 & 82,7 \\
\hline Sin información & 631 & 8,8 \\
\hline Total & 7.135 & 100 \\
\hline \multicolumn{3}{|l|}{ Grupos de edad (años) } \\
\hline $0-4$ & 2.082 & 29,2 \\
\hline $5-9$ & 201 & 2,8 \\
\hline $10-14$ & 245 & 3,4 \\
\hline $15-19$ & 389 & 5,5 \\
\hline $20-24$ & 283 & 4,0 \\
\hline $25-29$ & 256 & 3,6 \\
\hline $30-34$ & 248 & 3,5 \\
\hline $35-39$ & 231 & 3,2 \\
\hline $40-44$ & 268 & 3,8 \\
\hline $45-49$ & 348 & 4,9 \\
\hline $50-54$ & 388 & 5,4 \\
\hline $55-59$ & 419 & 5,9 \\
\hline $60-64$ & 399 & 5,6 \\
\hline 65 y más & 1.378 & 19,3 \\
\hline Total & 7.135 & 100 \\
\hline \multicolumn{3}{|l|}{ Área de residencia } \\
\hline Cabecera municipal & 5.962 & 83,6 \\
\hline Centro poblado & 295 & 4,1 \\
\hline Rural disperso & 808 & 11,3 \\
\hline Sin información & 70 & 1,0 \\
\hline Total & 7.135 & 100 \\
\hline
\end{tabular}

Fuente: DANE, Estadísticas vitales. Colombia

* Educación media: incluye media académica, media técnica y normalista

** Educación superior: incluye técnica profesional, tecnológica, universitaria y posgrados

${ }_{* \star \star}$ Centro poblado: incluye inspección, corregimiento o caserío

Los resultados del análisis según el ente territorial de residencia, indicaron que Bogotá, Antioquia, Valle del Cauca, Cundinamarca, Santander, Barranquilla, 


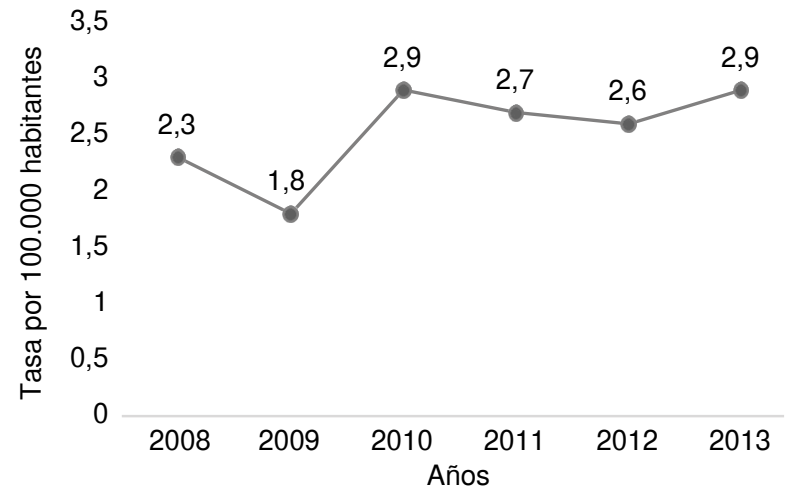

Figura 1. Tendencia de las tasas de mortalidad cruda por enfermedades huérfanas, Colombia, 2008-2013

Fuente: cálculo a partir de Estadísticas vitales, DANE
Cartagena, Tolima, Boyacá y Norte de Santander, presentaron enfermedades huérfanas con mayor frecuencia: en promedio, más de 40 casos en los seis años de estudio. La tasa de mortalidad nacional ajustada por edad durante los seis años de estudio, en promedio, fue de 2,6 por 100.000 personas, y por regiones, Bogotá aportó la tasa más alta $(20,0)$, presentando un aumento sostenido hasta el final del periodo. Las tasas promedio en las regiones de la Amazonia, la Orinoquia y la Pacífica fueron más bajas al compararse con las otras regiones. En el distrito especial de Bogotá, la tasa promedio fue de 20,0 muertes por 100.000, y en los departamentos de Cundinamarca y Sucre, de 21,5 y de 11,3, respectivamente (cuadro 5).

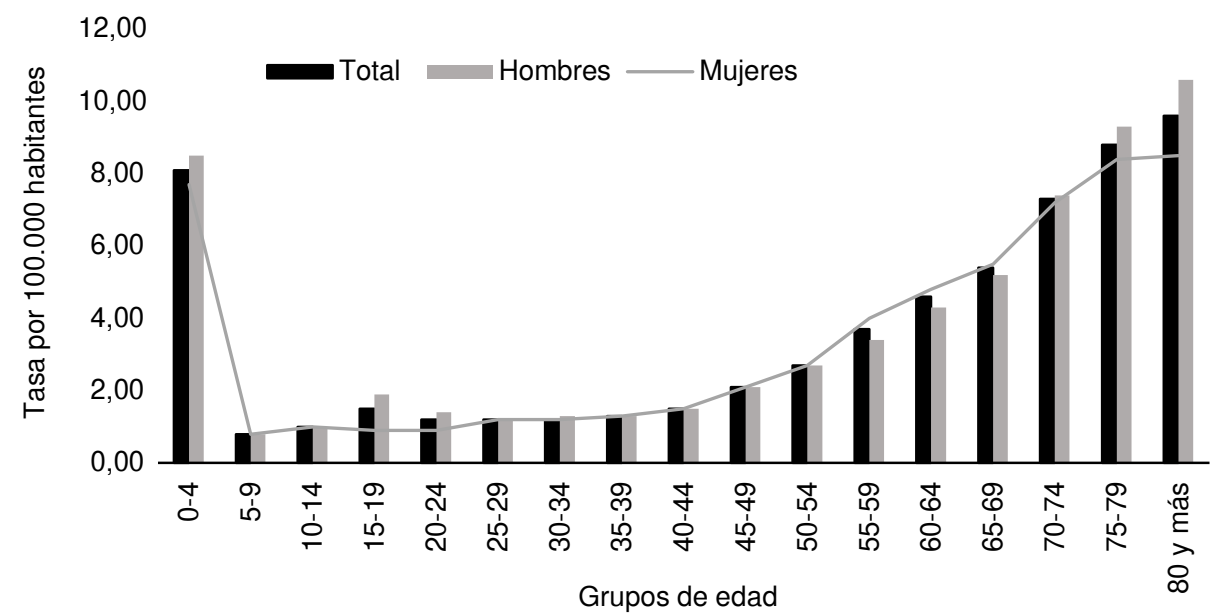

Figura 2. Tasas medias anuales específicas de enfermedades huérfanas, según sexo y grupos de edad, Colombia, 2008-2013 Fuente: cálculo a partir de Estadísticas vitales, DANE

Cuadro 3. Principales causas de mortalidad por enfermedades huérfanas en hombres, Colombia, 2008-2013

\begin{tabular}{|c|c|c|c|c|c|c|c|}
\hline \multirow[t]{2}{*}{ Enfermedad huérfana } & \multicolumn{7}{|c|}{ Tasa de mortalidad por 100.000 hombres } \\
\hline & 2008 & 2009 & 2010 & 2011 & 2012 & 2013 & Tasa media \\
\hline Leucemia linfoblástica aguda & 0,15 & 0 & 0,19 & 0,18 & 0,20 & 0,31 & 0,17 \\
\hline Distrofia muscular & 0,12 & 0,14 & 0,17 & 0,18 & 0,14 & 0,29 & 0,17 \\
\hline Displasia broncopulmonar originada en el periodo perinatal & 0,10 & 0,09 & 0,18 & 0,13 & 0,16 & 0,14 & 0,13 \\
\hline Esclerosis múltiple & 0,11 & 0,10 & 0,16 & 0,09 & 0,12 & 0,14 & 0,12 \\
\hline Síndrome de Guillain-Barre & 0,10 & 0,08 & 0,11 & 0,17 & 0,12 & 0,12 & 0,12 \\
\hline Gastrosquisis & 0,12 & 0,08 & 0,12 & 0,11 & 0,12 & 0,10 & 0,11 \\
\hline Leucemia mieloide aguda & 0 & 0 & 0,12 & 0,09 & 0,12 & 0 & 0,05 \\
\hline Anemia falciforme sin crisis & 0,06 & 0,04 & 0,06 & 0 & 0 & 0,08 & 0,04 \\
\hline Linfoma no Hodgkin, no especificado & 0 & 0 & 0,07 & 0,07 & 0,08 & 0,06 & 0,04 \\
\hline Deficiencia hereditaria del factor VIII & 0,05 & 0,04 & 0 & 0,06 & 0 & 0 & 0,03 \\
\hline Enfermedad de Hirschsprung & 0,04 & 0,04 & 0,07 & 0 & 0,06 & 0 & 0,03 \\
\hline Malformaciones congénitas múltiples, no clasificadas en otra parte & 0 & 0 & 0 & 0 & 0,05 & 0,06 & 0,02 \\
\hline Estenosis congénita de la válvula tricúspide & 0,05 & 0 & 0 & 0 & 0 & 0 & 0,01 \\
\hline Onfalocele & 0 & 0,04 & 0 & 0,06 & 0 & 0 & 0,01 \\
\hline Agranulocitosis & 0 & 0 & 0 & 0 & 0 & 0,05 & 0,00 \\
\hline Otras enfermedades huérfanas & 1,57 & 1,20 & 1,97 & 1,72 & 1,60 & 1,65 & 1,61 \\
\hline
\end{tabular}

Fuente: DANE. Estadísticas vitales, Colombia 
Cuadro 4. Principales causas de mortalidad por enfermedades huérfanas en mujeres, Colombia, 2008-2013

\begin{tabular}{|c|c|c|c|c|c|c|c|}
\hline \multirow{2}{*}{ Enfermedad huérfana } & \multicolumn{7}{|c|}{ Tasa de mortalidad por 100.000 mujeres } \\
\hline & 2008 & 2009 & 2010 & 2011 & 2012 & 2013 & Tasa media \\
\hline Esclerosis múltiple & 0,17 & 0,15 & 0,15 & 0,18 & 0,17 & 0,15 & 0,16 \\
\hline Leucemia linfoblástica aguda & 0,16 & 0,00 & 0,15 & 0,18 & 0,17 & 0,17 & 0,14 \\
\hline Gastrosquisis & 0,09 & 0,07 & 0,10 & 0,09 & 0,09 & 0,10 & 0,09 \\
\hline Displasia broncopulmonar originada en el periodo perinatal & 0,00 & 0,08 & 0,11 & 0,11 & 0,14 & 0,10 & 0,09 \\
\hline Síndrome de Guillain-Barré & 0,05 & 0,07 & 0,09 & 0,09 & 0,11 & 0,10 & 0,08 \\
\hline Leucemia mieloide aguda & 0,05 & 0 & 0,10 & 0,14 & 0,10 & 0,05 & 0,07 \\
\hline Anemia falciforme sin crisis & 0,06 & 0 & 0,07 & 0,06 & 0,07 & 0,06 & 0,05 \\
\hline Enfermedad de Alzheimer de comienzo temprano & 0 & 0 & 0 & 0,05 & 0 & 0 & 0,05 \\
\hline Miastenia gravis & 0,05 & 0,05 & 0,00 & 0 & 0,08 & 0 & 0,03 \\
\hline Linfoma no Hodgkin, no especificado & 0 & 0 & 0,06 & 0,05 & 0,07 & 0 & 0,03 \\
\hline Anomalía de Ebstein & 0,05 & 0 & 0,05 & 0 & 0 & 0 & 0,02 \\
\hline Atresia de los conductos biliares & 0,05 & 0,05 & 0 & 0 & 0 & 0 & 0,02 \\
\hline Hepatitis autoinmunitaria & 0 & 0 & 0,06 & 0 & 0,07 & 0 & 0,02 \\
\hline Poliomiositis & 0,05 & 0 & 0 & 0 & 0 & 0 & 0,01 \\
\hline Holoprosencefalia & 0 & 0,07 & 0 & 0 & 0 & 0 & 0,01 \\
\hline Distrofia muscular & 0 & 0,04 & 0 & 0 & 0 & 0,06 & 0,01 \\
\hline Malformaciones congénitas múltiples, no clasificadas en otra parte & 0 & 0 & 0 & 0,00 & 0 & 0,08 & 0,01 \\
\hline Síndrome de dificultad respiratoria del recién nacido & 0 & 0,04 & 0 & 0 & 0 & 0 & 0,00 \\
\hline Agranulocitosis & 0 & 0,04 & 0 & 0 & 0 & 0 & 0,00 \\
\hline Lupus eritematoso con compromiso de órganos o sistemas & 0 & 0 & 0 & 0,05 & 0 & 0 & 0,00 \\
\hline Enfermedad de Huntington & 0 & 0 & 0 & 0,00 & 0 & 0,06 & 0,00 \\
\hline Otras enfermedades huérfanas & 1,49 & 1,15 & 1,81 & 1,72 & 1,55 & 1,84 & 1,59 \\
\hline
\end{tabular}

Fuente: DANE. Estadísticas vitales, Colombia

\section{Discusión}

Entre el 2008 y el 2013, se presentaron 7.135 defunciones en Colombia, en las cuales alguna causa consignada de defunción fue por enfermedades huérfanas. No obstante, esta cifra podría ser mayor de lo que permiten ver los hallazgos presentados en este trabajo, en primer lugar, debido a la dificultad para el diagnóstico dada la rareza de muchas de estas enfermedades $y$, por otro lado, al desconocimiento del subregistro real pues un número importante de personas que las padecen mueren por complicaciones relacionadas y dichos fallecimientos comúnmente se clasifican de manera inadecuada en el certificado de defunción. Sin embargo, procurando limitar este inconveniente, la depuración de las bases de datos incluyó el diagnóstico de enfermedades huérfanas en cualquiera de las causas de defunción y no solo en las causas básicas.

En el análisis de las características sociales y demográficas de los fallecimientos, se observó un comportamiento similar en ambos sexos. En general, la mayor proporción de muertes, en su mayoría de origen genético, se registró en la población masculina de todos los grupos de edad, sobre todo en los menores de cinco años y en adultos mayores de 65 años. Esta tendencia concuerda con los datos oficiales sobre la morbilidad en Colombia por enfermedades huérfanas o raras, notificados al Sistema Vigilancia en Salud Publica en el 2016, los cuales documentan un comportamiento similar que ha venido aumentando de forma rápida y más acentuada en los hombres (10). En este contexto, dichas enfermedades adquieren una gran importancia para la salud pública, ya que estas cifras reflejan la gran carga de morbilidad y mortalidad prematura 0 , incluso, antes de cumplir la esperanza de vida promedio (70 años), lo cual produce, además de un alto grado de discapacidad y dependencia, un deterioro significativo de la calidad de vida de los afectados $(4,11)$.

En cuanto a nivel educativo, se observó una mayor proporción de personas fallecidas sin escolaridad y con básica primaria, lo cual podría deberse, entre otras causas, al carácter crónico y debilitante de las enfermedades, el compromiso de las habilidades físicas y mentales (12), las implicaciones o consecuencias sociales que pueden conducir al estigma, el aislamiento y la exclusión social (4), y a discapacidades coexistentes que acarrean múltiples consecuencias funcionales (la denominada multidiscapacidad o pluridiscapacidad); estas discapacidades refuerzan la sensación de aislamiento, pueden ser una fuente de discriminación y reducir o destruir oportunidades educativas (5). 
Cuadro 5. Tasas de mortalidad por enfermedades huérfanas, ajustadas por edad, según regiones y departamentos de residencia habitual, Colombia, 2008-2013

\begin{tabular}{|c|c|c|c|c|c|c|c|c|}
\hline \multirow[t]{2}{*}{ Regiones } & \multirow[t]{2}{*}{ Ente territorial } & \multicolumn{6}{|c|}{$\begin{array}{c}\text { Tasa ajustada de mortalidad por } 100.000 \\
\text { habitantes/año }\end{array}$} & \multirow[t]{2}{*}{$\begin{array}{c}\text { Promedio naciona } \\
\text { y regional }\end{array}$} \\
\hline & & 2008 & 2009 & 2010 & 2011 & 2102 & 2013 & \\
\hline \multirow[t]{4}{*}{ Colombia } & & 2,4 & 1,9 & 2,9 & 2,7 & 2,6 & 2,8 & 2,6 \\
\hline & Bogotá, D.C & 15,0 & 13,3 & 24,3 & 22,4 & 22,2 & 23,0 & 20 \\
\hline & Amazonas & 1,1 & 0,0 & 3,1 & 0,0 & 7,9 & 2,0 & 2,3 \\
\hline & Caquetá & 1,7 & 1,1 & 2,7 & 2,7 & 2,7 & 3,3 & 2,4 \\
\hline \multirow[t]{6}{*}{ Amazonia } & Guainía & 4,1 & 0,0 & 0,0 & 0,0 & 0,0 & 2,0 & 1,0 \\
\hline & Guaviare & 1,5 & 2,3 & 0,8 & 1,2 & 2,8 & 0,0 & 1,4 \\
\hline & Putumayo & 0,8 & 0,0 & 1,2 & 1,0 & 0,6 & 1,6 & 0,9 \\
\hline & Vaupés & 0,0 & 1,7 & 2,0 & 0,0 & 0,0 & 0,0 & 0,6 \\
\hline & Total & 1,5 & 0,8 & 1,6 & 0,8 & 2,3 & 1,5 & 1,4 \\
\hline & Arauca & 0,5 & 0,2 & 0,2 & 0,1 & 0,5 & 0,4 & 0,3 \\
\hline \multirow[t]{9}{*}{ Orinoquia } & Casanare & 0,7 & 0,6 & 0,7 & 1,5 & 0,6 & 1,0 & 0,9 \\
\hline & Meta & 1,2 & 2,3 & 2,6 & 3,3 & 3,2 & 2,7 & 2,5 \\
\hline & Vichada & 0,0 & 0,0 & 5,4 & 0,0 & 1,1 & 1,2 & 1,3 \\
\hline & Total & 0,6 & 0,8 & 2,2 & 1,2 & 1,3 & 1,3 & 1,2 \\
\hline & Atlántico & 8,9 & 7,5 & 10,0 & 7,1 & 7,6 & 11,6 & 8,8 \\
\hline & Barranquilla & 0,76 & 0,78 & 0,58 & 0,60 & 0,61 & 0,64 & 0,6 \\
\hline & Bolívar & 2,3 & 0,9 & 1,9 & 0,9 & 1,6 & 1,8 & 1,6 \\
\hline & Cartagena & 3,2 & 1,8 & 2,5 & 3,4 & 3,0 & 2,9 & 2,8 \\
\hline & Cesar & 2,4 & 2,3 & 3,2 & 2,5 & 2,6 & 3,1 & 2,7 \\
\hline \multirow[t]{9}{*}{ Caribe } & Córdoba & 6,1 & 4,6 & 6,6 & 6,1 & 5,9 & 5,8 & 5,9 \\
\hline & La Guajira & 2,5 & 1,1 & 2,1 & 2,9 & 0,9 & 2,3 & 2,0 \\
\hline & Magdalena & 1,2 & 1,1 & 1,8 & 15,3 & 0,7 & 2,0 & 3,7 \\
\hline & Santa Marta & 1,8 & 1,5 & 0,6 & 2,2 & 1,5 & 2,6 & 1,7 \\
\hline & Sucre & 10,2 & 10,8 & 13,2 & 10,2 & 13,2 & 10,2 & 11,3 \\
\hline & San Andrés y Providencia & 0,1 & 0,0 & 0,1 & 0,1 & 0,1 & 0,0 & 0,1 \\
\hline & Total & 3,6 & 3,0 & 3,9 & 4,6 & 3,4 & 3,9 & 3,7 \\
\hline & Buenaventura & 2,3 & 1,4 & 2,5 & 0,8 & 2,2 & 1,0 & 1,7 \\
\hline & Cauca & 1,1 & 1,1 & 2,3 & 1,9 & 1,5 & 1,7 & 1,6 \\
\hline \multirow[t]{9}{*}{ Pacífica } & Chocó & 0,3 & 0,3 & 0,3 & 0,4 & 0,5 & 0,5 & 0,4 \\
\hline & Nariño & 1,2 & 1,5 & 1,6 & 1,8 & 1,9 & 2,2 & 1,7 \\
\hline & Valle del Cauca & 2,9 & 2,3 & 3,8 & 3,1 & 2,7 & 3,0 & 3 \\
\hline & Total & 1,6 & 1,3 & 2,1 & 1,6 & 1,8 & 1,7 & 1,7 \\
\hline & Antioquia & 2,4 & 1,7 & 2,6 & 2,5 & 2,4 & 2,9 & 2,5 \\
\hline & Boyacá & 1,8 & 0,9 & 3,2 & 2,7 & 3,0 & 1,9 & 2,2 \\
\hline & Caldas & 3,4 & 2,1 & 2,3 & 2,9 & 3,3 & 2,9 & 2,8 \\
\hline & Cundinamarca & 17,0 & 12,5 & 30,4 & 28,1 & 20,6 & 20,5 & 21,5 \\
\hline & Huila & 2,5 & 1,7 & 3,0 & 3,6 & 1,9 & 2,4 & 2,5 \\
\hline \multirow[t]{6}{*}{ Andina } & Norte de Santander & 2,5 & 1,0 & 2,4 & 1,9 & 2,3 & 3,2 & 2,2 \\
\hline & Quindío & 4,1 & 2,1 & 4,2 & 3,2 & 2,6 & 2,2 & 3,1 \\
\hline & Risaralda & 2,5 & 2,3 & 1,9 & 2,4 & 2,8 & 2,6 & 2,4 \\
\hline & Santander & 10,2 & 10,8 & 13,2 & 10,2 & 13,2 & 10,2 & 11,3 \\
\hline & Tolima & 2,1 & 1,3 & 3,2 & 2,9 & 3,3 & 2,6 & 2,6 \\
\hline & Total & 4,9 & 3,7 & 6,7 & 6,0 & 5,5 & 5,1 & 5,3 \\
\hline
\end{tabular}

Fuente: DANE. Estadísticas vitales, Colombia

La mayoría de los fallecidos estaban afiliados al régimen contributivo del SGSSS y, habitualmente, residían en las cabeceras municipales, información que concuerda con la reportada en el censo de 2013 (13).

Si bien el 82,7\% de las personas fallecidas se clasificaba en la categoría de "otros grupos étnicos", en este estudio se encontró que la anemia de células falciformes -0 drepanocitosis- fue la principal causa de muerte de la población que se reconocía como mulata, afrocolombiana o afrodescendiente. Esto coincide con otros estudios sobre esta enfermedad en cinco estados de Brasil (14) y en áreas con población negra en Colombia, en los cuales se determinó una correlación significativa entre la frecuencia de este tipo de hemoglobinopatías y la frecuencia de autorreconocimiento como afrocolombiano (15). 
Al analizar la mortalidad global por las enfermedades huérfanas priorizadas, las tasas crudas presentaron una tendencia al aumento en los seis años de estudio, lo cual podría deberse al fortalecimiento del sistema de información de las estadísticas vitales por la promulgación de la Ley 1392 de 2010, cuyo objeto reconoce que las enfermedades huérfanas representan un problema de especial interés en salud pública; además, en el artículo 14 especifica que, para cumplir con las funciones de inspección, vigilancia y control, la Superintendencia Nacional de Salud ejercerá sus funciones teniendo como base diferentes estándares, entre los cuales está vigilar que los actores del sistema garanticen la producción de los datos con calidad, cobertura, pertinencia, oportunidad y transparencia (6).

El análisis de las tasas de mortalidad por las enfermedades huérfanas en grupos, se convierte en una limitación, ya que el impacto sobre la esperanza de vida varía mucho de una enfermedad a otra. Algunas causan la muerte al nacer, muchas son degenerativas o amenazan la vida, mientras que otras son compatibles con una vida normal si se diagnostican a tiempo y se tratan adecuadamente (4).

Según el sexo, las tasas de mortalidad presentaron un comportamiento similar, pero fueron mayores en las edades extremas de la vida. La alta mortalidad en menores de cinco años podría explicarse porque muchas de las estas enfermedades son de origen genético $(4,12)$ y el $50 \%$ de ellas se inician en la infancia (4).

La tendencia al aumento en la mortalidad a partir de los 65 años en ambos sexos, indica que muchas de las personas diagnosticadas con una de estas enfermedades no fallecen en edades tempranas. Sin embargo, no hay información que permita verificar la calidad de vida de aquellas que superaron los 65 años, ya que la mayoría de dichas enfermedades afectan más de un órgano vital, tienen un curso clínico crónico, son progresivamente debilitantes (16) y, a veces, comprometen la autonomía del enfermo (4).

Las causas de defunción no difirieron notoriamente según el sexo; las leucemias continúan siendo la primera causa de muerte en menores de 15 años, con predominio de la variante linfoblástica en la población masculina. Esta tendencia coincide con lo observado en Latinoamérica y con los datos oficiales del Instituto Nacional de Cancerología del 2008, que indican que cerca del $50 \%$ de las muertes por cáncer pediátrico se deben a leucemias agudas (17-19). Además de ser una enorme carga en términos de costos financieros y sociales, esto es un indicador trazador del acceso y la calidad de la atención médica especializada para garantizar el manejo integral que requiere el niño con cáncer (20).

La mayor tasa de mortalidad debida a displasia broncopulmonar se registró en neonatos y, la debida a gastrosquisis, en hombres. Un estudio realizado en Bogotá indicó que nacer en una institución con baja mortalidad neonatal disminuía el riesgo de muerte (odds ratio, $\mathrm{OR}=0,308 ; \mathrm{IC}_{95 \%} 0,129-0,736$ ), pero incrementaba el de displasia broncopulmonar moderada a grave (21). En cuanto a la gastrosquisis, en un estudio en un hospital de tercer nivel en Cali, se documentó que el $1 \%$ de los mortinatos entre el 2004 y el 2006 habían presentado gastrosquisis (22). Sin embargo, otro estudio en el mismo lugar indicó que este diagnóstico no era el más frecuente entre los nacidos con defectos congénitos en Cali entre 2011 y 2012 (23).

La información de las tasas de mortalidad por tipo de enfermedades huérfanas y sexo, indicó que algunas enfermedades presentaban altas tasas de mortalidad únicamente en hombres, como la deficiencia congénita del factor VIII, la enfermedad de Hirschsprung, la estenosis congénita de la válvula tricúspide y el exónfalos. Se revisó en Orphanet si estas enfermedades solían ser más frecuentes en un sexo específico, sin encontrar información al respecto (24).

La esclerosis múltiple se situó entre las enfermedades huérfanas con mayor tasa de mortalidad en ambos sexos, y fue la mayor en mujeres, lo cual concuerda con lo reportado en Venezuela entre 1988 y 1998 (25), Ecuador (26), España (27) y Colombia (28).

La distrofia muscular fue una de las enfermedades huérfanas con altas tasas de mortalidad, principalmente en hombres. Se ha documentado que, específicamente, la distrofia muscular de Duchenne, afecta principalmente al sexo masculino, con una incidencia de 1 por cada 3.300 nacimientos de varones (24), no obstante, no es posible determinar con esta fuente de información si las distrofias eran de este tipo específico.

El síndrome de Guillain-Barré también presentó una constante en las tasas de mortalidad principales en los seis años de estudio y en ambos sexos; de acuerdo con el informe de la vigilancia en salud 
pública de morbilidad por enfermedades huérfanas en Colombia en 2016, esta fue la enfermedad más frecuente (11).

El patrón de las defunciones debidas a este grupo enfermedades presentó diferencias importantes según las regiones. Las tasas ajustadas de mortalidad fueron mayores en Bogotá, en la región Andina y en la región Caribe, lo que puede atribuirse, entre otras razones, a que los pacientes diagnosticados con alguna enfermedad huérfana, y especialmente aquellos afectados por sus complicaciones, requieren cuidados médicos en instituciones muy especializadas y estos centros de atención están ubicados generalmente en las principales ciudades capitales del país y las personas pueden acceder a este tipo servicios por medio del sistema de referencia y contrarreferencia que ofrece el modelo de salud actual (29).

De acuerdo con lo reportado por el Ministerio de Salud y Protección Social en el análisis de situación de salud de 2014, la mortalidad por enfermedades crónicas en el país se concentra principalmente en la región Andina, por lo cual los perfiles de mortalidad regional plantean la necesidad de formular respuestas diferenciadas entre las regiones del país, según la afectación de los factores determinantes de la salud. Las intervenciones deben adaptarse a las condiciones de servicios, a las características de la demanda, y a la capacidad en recursos humanos y tecnológicos en cada territorio (30).

Los departamentos que registraron muertes por enfermedades huérfanas con mayor frecuencia, coinciden parcialmente con lo reportado por el Sistema Integral de Información de la Protección Social (SISPRO) con base en el censo elaborado en el 2013, cuando las mayores frecuencias se presentaron en Bogotá, Antioquia, Valle del Cauca, Santander, Atlántico, Cundinamarca, Bolívar, Risaralda, Caldas y Tolima (13).

Según este mismo informe, Bogotá, Cundinamarca, Sucre, Santander, Atlántico y Córdoba registraron las mayores tasas de mortalidad. Al comparar esta información con las prevalencias de enfermedades huérfanas (morbilidad) reportadas por la Cuenta de Alto Costo en 2013 (13), se observa que en las entidades territoriales como Sucre y Córdoba es mayor el número de personas que mueren por estas causas, lo cual podría deberse a deficiencias en la red de prestación de servicios para el diagnóstico oportuno y el tratamiento continuo de estos pacientes. Esta información indica la necesidad de estudios enfocados en la calidad de los servicios integrales para este grupo de enfermedades, en especial, en estos departamentos.

Las limitaciones del presente estudio radican principalmente en la fuente de información. En primer lugar, la cobertura de las estadísticas vitales no se ha logrado en todos los municipios de Colombia, dificultad que persiste tanto en el área urbana, por la falta de sistematización, como en la rural de las regiones más apartadas del país; además, no se dispone de otras fuentes de información secundaria y de calidad para comprobar la correspondencia (cruce de bases de datos) y, así, identificar las muertes que no fueron incluidas en la base del DANE. En segundo lugar, están las limitaciones relacionadas con la completitud y la validación de las variables del estudio, así como la mala codificación debido a que se registran eventos derivados de las complicaciones o de las enfermedades que se consideran en el diagnóstico diferencial.

A pesar de dichas limitaciones, el presente estudio es el primero en que se analiza el comportamiento de la mortalidad por las enfermedades huérfanas en su conjunto, priorizadas en el marco de la Resolución 2048 de 2015 del Ministerio de Salud y Protección Social, y puede ser la base para llevar a cabo nuevas investigaciones que permitan explorar diferentes metodologías de análisis, ampliar el conocimiento y priorizar aquellas regiones en donde se deberían evaluar las intervenciones actuales de salud pública, que puedan orientar a desarrollar mejores políticas y planes de prevención, atención y tratamiento que permitan mejorar la calidad de vida de las personas que padecen alguna de estas enfermedades y de sus familias.

Se concluyó que la mortalidad por enfermedades huérfanas priorizadas y analizadas en conjunto, ha mostrado una tendencia general a aumentar en el país. Si bien este comportamiento fue similar en ambos sexos, es importante mencionar que la población masculina de todas las edades registró las mayores tasas de mortalidad, principalmente por leucemia linfoblástica aguda, distrofia muscular, displasia broncopulmonar originada en el periodo perinatal, esclerosis múltiple, síndrome de Guillain-Barré y gastrosquisis. En las mujeres, la esclerosis múltiple fue la primera causa de muerte, seguida de leucemia linfoide aguda, gastrosquisis, displasia broncopulmonar originada en el periodo perinatal, síndrome de Guillain-Barré y leucemia mieloide aguda. 
La distribución geográfica ha sido heterogénea, concentrándose la mayor proporción de las muertes en Bogotá, y las regiones Andina y Caribe. El distrito especial de Bogotá y los departamentos de Cundinamarca, Sucre, Santander, Atlántico, Córdoba, Magdalena, Quindío, Caldas, Tolima, Huila y Antioquia registraron tasas de mortalidad (ajustadas por edad) mayores que la nacional.

\section{Agradecimientos}

Al Observatorio Nacional de Salud del Instituto Nacional de Salud, por facilitar las bases de datos con las cifras oficiales consolidadas a partir de las estadísticas vitales del Departamento Administrativo Nacional de Estadísticas (DANE) para el periodo de 2008 a 2013.

\section{Conflicto de intereses}

Los autores declaran no tener conflicto de intereses en relación con la preparación y publicación de este trabajo.

\section{Financiación}

No se recibió financiación para la elaboración de este artículo.

\section{Referencias}

1. Ministerio de Salud y Protección Social. Enfermedades huérfanas. Fecha de consulta: 19 de agosto de 2017. Disponible en: https://www.minsalud.gov.co/salud/publica/ PENT/Paginas/enfermedades-huerfanas.aspx

2. Instituto Nacional de Salud. Protocolo de vigilancia de enfermedades huérfanas-raras. Fecha de consulta: 9 de febrero de 2017. Disponible en: http://www.ins.gov.co/lineasde-accion/Subdireccion-Vigilancia/sivigila/Protocolos\%20 SIVIGILA/PRO\%20Enfermedades\%20hu\%C3\%A9rfanas\%20 y\%20raras.pdf

3. Congreso de la República de Colombia. Ley 1438 de 2011 (19 de enero) por medio del cual se reforma el Sistema General de Seguridad Social en Salud y se dictan otras disposiciones. Diario Oficial No. 47.957, 19 de enero de 2011. Bogotá: Congreso de Colombia; 2011.

4. EURORDIS European Organisation for rare diseases. Rare diseases: Understanding this public health priority. November 2005. Fecha de consulta: 9 de febrero de 2017. Disponible en: http://www.eurordis.org/IMG/pdf/princeps document-EN.pdf

5. Lugones M, Ramírez M. Enfermedades raras. Rev Cubana Med Gen Integr. 2012;28:340-50.

6. Congreso de la República de Colombia. Ley 1392 de 2010 (2 de julio) "por medio de la cual se reconocen las enfermedades huérfanas-raras como de especial interés y se adoptan normas tendientes a garantizar la protección social por parte del Estado colombiano a la población que padece enfermedades huérfanas-raras y sus cuidadores". Diario Oficial No. 47.758, 8 de julio de 2010. Bogotá: Congreso de Colombia; 2010.
7. Ministerio de Salud y Protección Social. Resolución 430 de 2013 "por la cual se define el listado de las enfermedades huérfanas-raras" del 20 de febrero de 2013. Bogotá: Ministerio de Salud y Protección Social; 2013.

8. Ministerio de Salud y Protección Social. Resolución 2048 de 2015 "por la cual se actualiza el listado de las enfermedades huérfanas y se define el número con el cual se identifica cada una de ellas en el sistema de información de pacientes con enfermedades huérfanas" del 9 de junio de 2015. Bogotá: Ministerio de Salud y Protección Social; 2015.

9. Organización Panamericana de la Salud. La estandarización: un método epidemiológico clásico para la comparación de tasas. Bol Epidemiol. 2002;23:1-5. Fecha de consulta: $1^{\circ}$ de marzo de 2017. Disponible en: http://www.bvsde.paho. org/bvsea/fulltext/estandar.pdf

10. Instituto Nacional de Salud. Informe del evento enfermedades huérfanas-raras, hasta el periodo epidemiológico trece, Colombia, 2016. Fecha de consulta: 6 de abril de 2017. Disponible en: http://www.bvsde.paho.org/bvsea/ fulltext/estandar.pdf

11. Avellaneda A, Izquierdo M, Torrent-Farnell J, Ramón JR. Enfermedades raras: enfermedades crónicas que requieren un nuevo enfoque sociosanitario. An Sist Sanit Navar. 2007;30:177-90.

12. Montserrat A, Waligóra J. The European Union policy in the field of rare diseases. Public Health Genomics. 2013; 16:268-77. https://doi.org/10.1159/000355930

13. Ministerio de Salud y Protección Social. Enfermedades huérfanas. Indicadores. Fecha de consulta: 19 de agosto de 2017. Disponible en: http://www.sispro.gov.co/Pages/ EnfermedadesHuerfanas/Indicadores.aspx

14. Oliveira GA, Pinto L, Trovó AB. Mortality by sickle cell disease in Brazil. Rev Bras Hematol Hemoter. 2017;39:526. https://doi.org/10.1016/j.bjhh.2016.09.008

15. Rosero MJ, Bermúdez AJ. Análisis de hemoglobinopatías en regiones afrocolombianas usando muestras de sangre seca de cordón umbilical. Acta Med Colomb. 2012;37: 118-24.

16. Posada M, Martín-Arribas C, Ramírez A, Villaverde A, Abaitua I. Enfermedades raras. Concepto, epidemiología y situación actual en España. An Sist Sanit Navar. 2008; 31(Supl.2):9-20

17. Vera AM, Pardo C, Duarte MC, Suárez A. Análisis de mortalidad por leucemia aguda pediátrica en el Instituto Nacional de Cancerología. Biomédica. 2012;32:355-64. https://doi.org/10.7705/biomedica.v32i3.691

18. Curado MP, Pontes T, Guerra-Yi ME, Cancela M. Leukemia mortality trends among children, adolescents, and young adults in Latin America. Rev Panam Salud Pública. 2011;29:96-102. https://doi.org/10.1590/S102049892011000200004

19. Instituto Nacional de Cancerología E.S.E. Cáncer en cifras: mortalidad por cáncer según primeras causas y sexo, tasas específicas por grupos de edad, Colombia, 2000-2008. Fecha de consulta: 16 de marzo de 2017. Disponible en: http://www.cancer.gov.co/documentos/ Mortalidad/Mortalidad\%20nacional\%20por\%20tipo\%20de\%20 cáncer\%20y\%20grupo\%20de\%20edad\%202000-2008.pdf 
20. Ministerio de Salud y Protección Social. Resolución 2590 de 2012 "por el cual se constituye el Sistema Integrado en Red y el Sistema Nacional de Información para el monitoreo, seguimiento y control de la atención del cáncer en los menores de 18 años, integrando la base de datos para la agilidad de la atención del menor con cáncer, el Registro Nacional de Cáncer Infantil y el número único nacional para los beneficiarios de la Ley 138820102 del 31 de agosto de 2012". Bogotá: Ministerio de Salud y Protección Social; 2012.

21. Ruiz J, Charpak N. Epidemia de displasia broncopulmonar: incidencia y factores asociados en una cohorte de niños prematuros en Bogotá, Colombia. Biomédica. 2014;34:2939. https://doi.org/10.7705/biomedica.v34i1.1463

22. Pachajoa H, Saldarriaga W, Isaza C. Gastrosquisis en un hospital de tercer nivel de la ciudad de Cali, Colombia durante el período marzo 2004 a febrero 2006. Colomb Med. 2008;39(Supl.2):35-40.

23. Ramírez J, Pachajoa H, Ariza Y, Isaza C, Saldarriaga W. Defectos congénitos en un hospital de tercer nivel en Cali, Colombia. Rev Chil Obstet Ginecol. 2015;80:442-9.

24. Orphanet. Portal de información de enfermedades raras y medicamentos huérfanos. Fecha de consulta: 29 de marzo de 2017. Disponible en: http://www.orpha.net/consor/cgibin/Disease.php?lng=EN.
25. Pérez C, López MH, Ortiz HN, Rodríguez M, Avilán JM. Tasa de mortalidad de la esclerosis múltiple en Venezuela según edad y género. Gac Méd Caracas. 2003;11:227-30.

26. Abada P, Pérez M, Castro E, Alarcón T, Santibáñez R, Díaz F. Prevalencia de esclerosis múltiple en Ecuador. Neurología. 2010;25:309-13. https://doi.org/10.1016/j.nrl. 2009.12.005

27. Bártulos M, Marzo ME, Ruiz E, Bravo Y. Estudio epidemiológico de la esclerosis múltiple en La Rioja. Neurología. 2015;30:552-60. https://doi.org/10.1016/j.nrl.2014.04.016

28. Jiménez C, Zarco L, Castañeda C, Otálora M, Martínez A, Rosselli D. Estado actual de la esclerosis múltiple en Colombia. Acta Neurol Colomb. 2015;31:385-90.

29. Congreso de la República de Colombia. Ley 100 de 1993 (23 de diciembre) "por la cual se crea el sistema de seguridad social integral y se dictan otras disposiciones". Diario Oficial No. 41.148 del 23 de diciembre de 1993. Bogotá: Congreso de Colombia; 1993.

30. Ministerio de Salud y Protección Social. Política de atención integral en salud. Fecha de consulta: 29 de marzo de 2017. Disponible en: https://www.minsalud.gov.co/sites/ rid/Lists/BibliotecaDigital/RIDE/DE/modelo-pais-2016.pdf 
Anexo 1. Homologación de códigos CIE-10 de enfermedades huérfanas

\begin{tabular}{|c|c|c|}
\hline $\begin{array}{l}\text { Nombre de la enfermedad huérfana } \\
\text { (Resolución } 2048 \text { de 2015) }\end{array}$ & $\begin{array}{l}\text { Código } \\
\text { CIE } 10\end{array}$ & Nombre CIE 10 \\
\hline Fiebre botonosa & A771 & Fiebre maculosa debido a Rickettsia conorii \\
\hline Enfermedad de Creutzfeldt-Jakob & A810 & Enfermedad de Creutzfeldt \\
\hline Amebiasis por amebas salvajes & $\mathrm{B} 601$ & Acantamebiasis \\
\hline Anisakiasis & B810 & Anisaquiasis \\
\hline Síndrome de Sézary & $\mathrm{C} 841$ & Enfermedad de Sézary \\
\hline Enfermedad de Letterer-Siwe & C960 & Enfermedad de Letterer \\
\hline Síndrome de Plummer-Vinson & D501 & Disfagia sideropénica \\
\hline Betatalasemia & D561 & Betatalasemia \\
\hline Drepanocitosis & D57 & Trastornos falciformes \\
\hline Esferocitosis hereditaria & D580 & Esferocitosis hereditaria \\
\hline Hemoglobinuria paroxística nocturna & D595 & Hemoglobinuria paroxística nocturna (marchiafava) \\
\hline Anemia de Fanconi & D610 & Anemia aplástica constitucional \\
\hline Anemia sideroblástica ligada al cromosoma $X$ & D640 & Anemia sideroblástica hereditaria \\
\hline Déficit congénito del factor VIII & D66 & Deficiencia hereditaria del factor VIII \\
\hline Déficit congénito del factor IX & D67 & Deficiencia hereditaria del factor IX \\
\hline Enfermedad de von Willebrand & D680 & Enfermedad de von Willebrand \\
\hline Déficit congénito del factor XI & D681 & Deficiencia hereditaria del factor XI \\
\hline Déficit congénito de fibrinógeno & D682 & Deficiencia hereditaria de otros factores de la coagulación \\
\hline Enfermedad de von Willebrand, adquirida & D684 & Deficiencia adquirida de factores de la coagulación \\
\hline Púrpura de Henoch-Schönlein & D690 & Púrpura alérgica \\
\hline Neutropenia cíclica & D70 & Agranulocitosis \\
\hline Inmunodeficiencia debida a déficit de CD25 & D812 & $\begin{array}{l}\text { Inmunodeficiencia combinada severa [IDCS] con cifra baja o } \\
\text { normal de linfocitos B }\end{array}$ \\
\hline $\begin{array}{l}\text { Inmunodeficiencia por expresión deficiente del } \\
\text { HLA de clase } 2\end{array}$ & D817 & $\begin{array}{l}\text { Deficiencia de la clase II del complejo de histocompatibilidad } \\
\text { mayor }\end{array}$ \\
\hline Síndrome de Wiskott-Aldrich & D820 & Síndrome de Wiskott \\
\hline Síndrome de hiper-IgE autosómico dominante & D824 & Síndrome de hiperinmunoglobulina e $[\mathrm{lg} E]$ \\
\hline Inmunodeficiencia común variable & D83 & Inmunodeficiencia variable común \\
\hline Sarcoidosis & D86 & Sarcoidosis \\
\hline Crioglobulinemia mixta & D891 & Crioglobulinemia \\
\hline Acromegalia & E220 & Acromegalia y gigantismo hipofisario \\
\hline $\begin{array}{l}\text { Síndrome de secreción inapropiada de } \\
\text { hormona antidiurética }\end{array}$ & E222 & Síndrome de secreción inapropiada de hormona antidiurética \\
\hline Síndrome de Cushing & E24 & Síndrome de Cushing \\
\hline Enfermedad de Cushing & E240 & Enfermedad de Cushing dependiente de la hipófisis \\
\hline Síndrome de insensibilidad a los andrógenos & E345 & Síndrome de resistencia androgénica \\
\hline Fenilcetonuria & E700 & Fenilcetonuria clásica \\
\hline Albinismo ocular sordera sensorial tardía & E703 & Albinismo \\
\hline $\begin{array}{l}\text { Trastornos del metabolismo de los ácidos } \\
\text { grasos }\end{array}$ & E713 & Trastornos del metabolismo de los ácidos grasos \\
\hline Galactosemia & E742 & Trastorno del metabolismo de la galactosa \\
\hline Lipofuscinosis neuronal ceroide tardía infantil & E754 & Lipofuscinosis ceroide neuronal \\
\hline Síndrome de Hurler & E760 & Mucopolisacaridosis tipo I \\
\hline Mucopolisacaridosis de tipo 2 & E761 & Mucopolisacaridosis tipo II \\
\hline Mucopolisacaridosis de tipo 7 & E762 & Otras mucopolisacaridosis \\
\hline Mucopolisacaridosis no especificada & E763 & Mucopolisacaridosis no especificada \\
\hline Mucolipidosis de tipo 2 & E770 & $\begin{array}{l}\text { Defectos en la modificación postraslacional de enzimas } \\
\text { lisosomales }\end{array}$ \\
\hline Síndrome de Lesch-Nyhan & E791 & Síndrome de Lesch \\
\hline Porfiria eritropoyética congénita & E800 & Porfiria eritropoyética hereditaria \\
\hline Síndrome de Crigler-Najjar & E805 & Síndrome de Crigler \\
\hline Fibrosis quística & E84 & Fibrosis quística \\
\hline
\end{tabular}




\begin{tabular}{|c|c|c|}
\hline Polineuropatia amiloide familiar & E851 & Amiloidosis heredofamiliar neurop[atica \\
\hline Amiloidosis secundaria & E853 & Amiloidosis sistémica secundaria \\
\hline $\begin{array}{l}\text { Lipodistrofia familiar parcial asociada con } \\
\text { mutaciones en PPARG }\end{array}$ & E881 & Lipodistrofia, no clasificada en otra parte \\
\hline Lipomatosis encefalocraneocutanea & E882 & Lipomatosis, no clasificada en otra parte \\
\hline Síndrome de Rett & F842 & Síndrome de Rett \\
\hline Enfermedad de Huntington & G10 & Enfermedad de Huntington \\
\hline Ataxia espinocerebelosa de tipo 29 & G11 & Ataxia hereditaria \\
\hline Ataxia de Friedreich & G111 & Ataxia cerebelosa de iniciación temprana \\
\hline $\begin{array}{l}\text { Paraplejia espástica autosómica dominante } \\
\text { de tipo } 10\end{array}$ & G114 & Paraplejia espástica hereditaria \\
\hline Atrofia muscular espinal proximal & G120 & Atrofia muscular espinal infantil, tipo I [Werdnig] \\
\hline Síndrome neuroléptico maligno & G210 & Síndrome neuroléptico maligno \\
\hline $\begin{array}{l}\text { Enfermedad de Alzheimer autosómica } \\
\text { dominante de aparición temprana }\end{array}$ & G300 & Enfermedad de Alzheimer de comienzo temprano \\
\hline Esclerosis múltiple & G35 & Esclerosis múltiple \\
\hline Enfermedad de Devic & G360 & Neuromielitis óptica [Devic] \\
\hline Enfermedad de Refsum & G601 & Enfermedad de Refsum \\
\hline Neuropatía axonal aguda motora y sensitiva & G610 & Síndrome de Guillain \\
\hline Miastenia grave & G700 & Miastenia gravis \\
\hline Síndromes miasténicos congénitos & G702 & Miastenia congénita o del desarrollo \\
\hline Distrofia facioescapulohumeral & G710 & Distrofia muscular \\
\hline Síndrome miasténico de Lambert-Eaton & G731 & Síndrome de Eaton \\
\hline Disautonomía familiar & G901 & Disautonomia familiar [síndrome de Riley] \\
\hline Siringomielia & G950 & Siringomielia y siringobulbia \\
\hline Atrofia óptica & $\mathrm{H} 472$ & Atrofia óptica \\
\hline Fiebre reumática & 101 & Fiebre reumática con complicación cardiaca \\
\hline Enfermedad de Rendu-Osler-Weber & 1780 & Telangiectasia hemorrágica hereditaria \\
\hline Síndrome de Budd-Chiari & 1820 & Síndrome de Budd \\
\hline Hernia diafragmática & K44 & Hernia diafragmática \\
\hline Enfermedad de Crohn & K50 & Enfermedades de Crohn [enteritis regional] \\
\hline Cirrosis biliar primaria & K743 & Cirrosis biliar primaria \\
\hline Hepatitis crónica autoinmunitaria & K754 & Hepatitis autoinmune \\
\hline Enfermedad veno-oclusiva hepática & K765 & Enfermedad veno \\
\hline Pénfigo vulgar & L100 & Pénfigo vulgar \\
\hline Pénfigo foliáceo & L102 & Pénfigo foliáceo \\
\hline Dermatosis pustulosa subcórnea & L131 & Dermatitis pustulosa subcorneal \\
\hline Reumatismo psoriásico & L405 & Artropatía psoriásica (m07.0 \\
\hline Pityriasis rubra pilaris & L440 & Pitiriasis rubra pilaris \\
\hline Urticaria solar & L563 & Urticaria solar \\
\hline Hipertricosis lanuginosa adquirida & L681 & Hipertricosis lanuginosa adquirida \\
\hline Esclerosis sistémica cutánea limitada & L940 & Escleroderma localizado [morfea] \\
\hline Enfermedad de Still del adulto & M061 & Enfermedad de Still de comienzo en el adulto \\
\hline Artritis juvenil idiopática de inicio sistémico & M082 & Artritis juvenil de comienzo generalizado \\
\hline Periarteritis nodosa & M300 & Poliarteritis nudosa \\
\hline Síndrome de Churg-Strauss & M301 & Poliarteritis con compromiso pulmonar [Churg] \\
\hline Dermatomiositis & M330 & Dermatomiositis juvenil \\
\hline Poliomiositis & M332 & Polimiositis \\
\hline Esclerosis sistémica cutánea difusa & M340 & Esclerosis sistémica progresiva \\
\hline Síndrome CREST & M341 & Síndrome CREST \\
\hline Fascitis eosinofílica & M354 & Fascitis difusa (eosinofílica) \\
\hline Fibrodisplasia osificante progresiva & M611 & Miositis osificante progresiva \\
\hline Enfermedad de Paget juvenil & M88 & Enfermedades de Paget de los huesos [osteítis deformante] \\
\hline Policondritis atrofiante & M941 & Policondritis recidivante \\
\hline Diabetes insípida nefrogénica & N251 & Diabetes insípida nefrógena \\
\hline
\end{tabular}




\begin{tabular}{|c|c|c|}
\hline Enfermedad de Dent & N258 & Otros trastornos resultantes de la función tubular renal alterada \\
\hline Displasia broncopulmonar & P271 & Displasia broncopulmonar originada en el periodo perinatal \\
\hline Síndrome de rubéola congénita & P350 & Síndrome de rubéola congénita \\
\hline Diabetes mellitus neonatal & P702 & Diabetes mellitus neonatal \\
\hline Craneoraquisquisis & Q001 & Craneorraquisquisis \\
\hline Holoprosencefalia & Q042 & Holoprosencefalia \\
\hline Aniridia & Q131 & Ausencia del iris \\
\hline Microtia & Q172 & Microtia \\
\hline Estenosis pulmonar valvular & Q221 & Estenosis congénita de la válvula pulmonar \\
\hline Atresia tricúspide & Q224 & Estenosis congénita de la válvula tricúspide \\
\hline Malformación de Ebstein & Q225 & Anomalía de Ebstein \\
\hline Atresia de coanas & Q300 & Atresia de las coanas \\
\hline Arrinia & Q301 & Agenesia o hipoplasia de la nariz \\
\hline Atresia de intestino delgado & Q41 & Ausencia, atresia y estenosis congénita del intestino delgado \\
\hline Atresia duodenal & Q410 & Ausencia, atresia y estenosis congénita del duodeno \\
\hline Triada de Currarino & Q42 & Ausencia, atresia y estenosis congénita del intestino grueso \\
\hline Enfermedad de Hirschsprung & Q431 & Enfermedad de Hirschsprung \\
\hline Atresia biliar & Q442 & Atresia de los conductos biliares \\
\hline Agenesia renal bilateral & Q601 & Agenesia renal, bilateral \\
\hline Plagiocefalia aislada & Q673 & Plagiocefalia \\
\hline Hemimelia tibial & Q725 & Defecto por reducción longitudinal de la tibia \\
\hline Hemimelia fibular & Q726 & Defecto por reducción longitudinal del peroné \\
\hline Síndrome de Klippel-Feil aislado & Q761 & Síndrome de Klippel \\
\hline Acondrogénesis & Q770 & Acondrogénesis \\
\hline Enanismo tanatofórico & Q771 & Enanismo tanatofórico \\
\hline Acondroplasia & Q774 & Acondroplasia \\
\hline Displasia espondilo-encondral & Q777 & Displasia espondiloepifisaria \\
\hline Osteogénesis imperfecta & Q780 & Osteogénesis imperfecta \\
\hline Encondromatosis & Q784 & Encondromatosis \\
\hline Onfalocele & Q792 & Exónfalos \\
\hline Gastrosquisis & Q793 & Gastrosquisis \\
\hline Síndrome de Ehlers-Danlos de tipo vascular & Q796 & Síndrome de Ehlers \\
\hline Ictiosis ligada al cromosoma $X$ & Q801 & Ictiosis ligada al cromosoma $\mathrm{x}$ \\
\hline Ictiosis lamelar & Q802 & Ictiosis lamelar \\
\hline Colestasis linfedema & Q820 & Linfedema hereditario \\
\hline Xeroderma pigmentoso & Q821 & Xeroderma pigmentoso \\
\hline Mastocitosis & Q822 & Mastocitosis \\
\hline Incontinentia pigmenti & Q823 & Incontinencia pigmentaria \\
\hline Esclerosis tuberosa & Q851 & Esclerosis tuberosa \\
\hline Síndrome de Marfan & Q874 & Síndrome de Marfan \\
\hline Trisomía 18 & Q910 & Trisomía 18, por falta de disyunción meiótica \\
\hline Trisomía 13 & Q914 & Trisomía 13, por falta de disyunción meiótica \\
\hline Duplicación 6p & Q923 & Trisomía parcial menor \\
\hline Cromosoma 1 en anillo & Q932 & Cromosoma reemplazado por anillo o dicéntrico \\
\hline Síndrome de Wolf-Hirschhorn & Q933 & Supresión del brazo corto del cromosoma 4 \\
\hline Monosomía 5p & Q934 & Supresión del brazo corto del cromosoma 5 \\
\hline Síndrome de Turner & Q96 & Síndrome de Turner \\
\hline Disgenesia gonadal anomalías múltiples & Q991 & Hermafrodita verdadero $46, x x$ \\
\hline
\end{tabular}

Fuente: Resolución 2048 de 2015. Clasificación Estadística Internacional de Enfermedades y Problemas Relacionados con la Salud, decima revisión. 